\title{
Circulatory support with larger volume intra-aortic balloon pump vs. standard volume or no-balloon pump during high-risk percutaneous coronary interventions. A randomised study
}

\author{
Aleksander Zeliaś ${ }^{1}$, Wojciech Zajdel², Krzysztof Malinowski ${ }^{3}$, Jolanta Geremek ${ }^{2}$, Krzysztof Żmudka² \\ ${ }^{1}$ Department of Cardiology and Cardiovascular Interventions, University Hospital, Krakow, Poland \\ ${ }^{2}$ Clinic of Interventional Cardiology, John Paul II Hospital, Krakow, Poland \\ ${ }^{3}$ Faculty of Health Sciences, Institute of Public Health, Jagiellonian University Medical College, Krakow, Poland
}

Adv Interv Cardiol 2020; 16, 1 (59): 30-40

DOI: https://doi.org/10.5114/aic.2020.93910

\begin{abstract}
A bstract
Introduction: Percutaneous coronary intervention in high-risk patients (HRPCI) is associated with increased risk of periprocedural complications such as hypotension and shock. Mechanical circulatory support devices may the bridge patient safely throughout the procedure and are often used in this setting.

Aim: We assessed the outcomes of patients subjected to $\mathrm{HRPCl}$ and supported with intra-aortic balloon pump (IABP) of larger volume (MEGA) compared to standard volume (STRD) or no balloon support at all (CTRL).

Material and methods: In this single-centre, open-label, randomised, controlled trial, $\mathrm{HRPCl}$ patients were randomly assigned to three groups: MEGA, STRD, and CTRL in a $1: 1: 1$ scheme. Screening failure patients were assigned to the registry (REG). Composite haemodynamic endpoint (CHEP) was assessed during the procedure and major adverse cardiac even (MACE)/safety endpoints up to 1-year follow-up (FU).

Results: A total of 36 patients were randomised (13 MEGA, 14 STRD, and 9 CTRL). The incidence of in-hospital MACE was observed in $23.1 \%$ of MEGA, $7.1 \%$ of STRD and $33.3 \%$ of CTRL $(p=0.25)$ patients; MACE at FU in $50.0 \%, 35.7 \%$, and $55.6 \%(p=0.61)$; major bleeding in $46.2 \%, 28.6 \%$, and $22.2 \%,(p=0.45)$; and CHEP in $15.4 \%, 50.0 \%$, and $44.4 \%$, respectively $(p=0.13)$. On per-treatment (PT) analysis (16 MEGA, 10 STRD, and 21 CTRL), including 11 patients from REG, in-hospital MACE was observed in $18.8 \%$ of MEGA, $10.0 \%$ of STRD, and $23.8 \%$ of CTRL ( $p=0.64)$ patients; MACE at FU in $53.3 \%, 20.0 \%$, and $57.1 \%(p=0.12)$; major bleeding in $37.5 \%, 20.0 \%$, and $33.3 \%(p=0.62)$; and CHEP in $15.5 \%, 50.0 \%$, and $52.4 \%$, respectively $(p=0.023)$.

Conclusions: Larger volume intra-aortic balloon pump might be effective at reducing haemodynamic instability during $\mathrm{HRPCI}$ without a statistically significant effect on safety endpoints or MACE.
\end{abstract}

Key words: instability, hypotension, circulatory support, intra-aortic balloon pump, high-risk percutaneous coronary intervention.

Su m m a ry

The inherent risk of percutanous coronary intervention performed in high-risk patients renders them vulnerable to complications. Nevertheless, there is no universal consensus on whether to use mechanical circulatory support devices in that setting, or on which kind of device is optimum in terms of safety and efficacy. The current study supports the use of wellknown intra-aortic balloon pump technology with increased balloon volume because it probably diminishes the risk of significant hypotension during high-risk percutaneous coronary interventions.

\section{Corresponding author:}

Aleksander Zeliaś MD, PhD, Department of Cardiology and Cardiovascular Interventions, University Hospital, 2 Jakubowskiego St, 30-688 Krakow, Poland, e-mail: aazelias@gmail.com

Received: 3.01.2020, accepted: 17.02.2020. 


\section{Introduction}

The recent developments in percutaneous coronary techniques allow for treatment of very sick patients with complex coronary anatomy, who are not suitable candidates for coronary artery bypass grafting (CABG) because of low ejection fraction (EF) and many comorbidities (socalled complex high risk and indicated patients - CHIP) [1]. This is invariably associated with increased risk of acute cardiac decompensation during the procedure, it explains the growing interest in short-term mechanical circulatory support devices (MCS). One of the bestknown examples of these is intra-aortic balloon pump (IABP), which was introduced to clinical practice in the late 1960s [2]. Its postulated mechanism of action is the augmentation of diastolic aortic pressure and reduction of left ventricular systolic and diastolic pressures, leading to increased coronary blood flow and decreased heart work, which is accompanied by a small increase in cardiac output and index. Despite controversies generated by negative results of randomized controlled trials (RCTs), especially lack of mortality benefit in acute myocardial infarction (AMI) complicated by cardiogenic shock (CS) in the IABP-SHOCK-II trail [3], failure to reduce major adverse cardiac even (MACE) in high-risk percutaneous coronary interventions $(\mathrm{HRPCl})$ patients in Balloon pump-assisted Coronary Intervention Study (BCIS-1) [4], and the inability of myocardial preservation in anterior $\mathrm{AMI}$ in CRISP AMI studies [5], IABP is still widely used by many practitioners worldwide as a valid alternative to much more expensive (Impella, Abiomed, Danvers, MA) or invasive (ECMO) devices, which is supported by the latest American guidelines with a $\mathrm{llb} / \mathrm{C}$ class recommendation (unfortunately there are no European guidelines) [6]. The latest modifications of the balloon, i.e. its increased volume, direct pressure measurement by light transmission, and improved synchronisation with cardiac cycle, make it probably more effective [7].

\section{Aim}

The present study was designed to compare, in a randomised fashion, outcomes of patients subjected to $\mathrm{HRPCl}$ and supported with IABP of larger volume (MEGA) compared to standard volume (STRD) or no balloon support at all (CTRL).

\section{Material and methods}

The study recruited patients scheduled for $\mathrm{HRPCl}$ after heart team consultation, according to clinical (not suitable candidates for surgery), echocardiographic (EF at or below 35\%), and angiographic criteria (unprotected left main (LM), multivessel disease (MVD), or last-remaining vessel or significant left main lesion with the right coronary artery occluded - the last two regardless of EF). The exclusion criteria were CS, acute coronary syndrome
(ACS) $<48 \mathrm{~h}$, aortic dissection or severe aortic regurgitation, significant peripheral artery disease, acute stroke, or contraindications for dual antiplatelet therapy (DAPT).

The patients were randomised in a $1: 1: 1$ fashion with randomly permuted blocks using web-based randomisation tool [8] to support with a larger volume (MEGA: $50 \mathrm{ml}>162 \mathrm{~cm}$ and $40 \mathrm{ml}<162 \mathrm{~cm}$ ), standard volume (STRD: $40 \mathrm{ml}>162 \mathrm{~cm}$ and $34 \mathrm{ml}<162 \mathrm{~cm}$ ), or no balloon control group (CTRL). In patients allocated to balloon treatment the maximum balloon inflation volume and $1: 1$ ratio of support was used throughout the procedure. Before $\mathrm{PCl}$ the right and left heart catheterisation (using Pulmonary Artery Catheter (PAC) via femoral or antecubital fossa vein and Pigtail catheter via femoral artery, respectively) was done with the assessment of intra-cardiac chamber pressures (including left ventricle systolic and diastolic pressures, pulmonary artery wedge pressure (PCWP), left ventricle pressure/time ratio (dP/dt), cardiac output (CO), and cardiac index $(\mathrm{Cl})$ by Fick principle). All $\mathrm{PCl}$ were performed by two experienced operators in the Interventional Cardiology Clinic of John Paul II Hospital in Krakow between March 2016 and October 2017, with the aim of DES implantation and complete revascularisation according to preprocedural viability testing. The arterial access was either femoral or radial, preferably with $7 \mathrm{Fr}$ sheaths. Coronary rotational atherectomy and intravascular ultrasound (IVUS) were used as needed. The invasive arterial pressure was constantly recorded by a separate line (usually radial). Procedural success was defined as residual stenosis of less than $30 \%$, TIMI 3 flow, and no major complications. Unfractionated heparin (70-100 U/kg) and DAPT were used (ticagrelor in ACS patients, clopidogrel in stable coronary artery disease or when longterm anticoagulation was indicated) as well as optimal medical therapy according to 2014 ESC/EACTS guidelines of myocardial revascularisation [9].

We recorded standard clinical, laboratory, angiographic, and echocardiographic parameters. We assessed symptoms (CCS and NYHA), in hospital and one-year follow-up MACE (deaths, AMI or ischaemia driven revascularisation, exacerbation of chronic heart failure, or stroke), haemorrhagic (major $(\geq 3)$ and minor $(<3)$ using BARC scale [10]), renal, and vascular complications. To define $\mathrm{MI}$, we used the BCIS-1 definition [11]: periprocedural $\mathrm{MI}$ (up to $72 \mathrm{~h}$ post intervention) was diagnosed when creatinine kinase $M B(C K-M B)$ increased more than $3 x$ in patients with normal CK-MB at baseline or $>1.5 \times$ when they were already elevated. After $72 \mathrm{~h}$ the standard ESC $\left(4^{\text {th }}\right)$ definition of MI was used [12]. For clinical risk assessment we used Euroscore II [13], for angiographic complexity and risk evaluation Syntax Score [14] and BCIS-1 Jeopardy Score [11]. All patients were followed up for 12 months after $\mathrm{PCl}$.

For the purpose of this study a new haemodynamic composite end point was defined. First, we introduced 
a novel hypotension index - hypotension load $(\mathrm{HL})$, which was calculated according to the following algorithm:

1) arterial pressure values were sampled in 10-second intervals,

2) for each minute interval a mean arterial pressure (MAP) value was calculated,

3) before the $\mathrm{PCl}$ the patient-specific arterial hypotension threshold (PSAHT) was set by calculating for a minimum of 5 min average MAP minus two standard deviations,

4) if, during $\mathrm{PCl}$, for a given minute, the MAP value was below PSAHT, then a hypotension episode was considered to be occurring in this minute, and the difference between PSAHT and MAP was calculated,

5) $\mathrm{HL}$ is the sum of (PSAHT-MAP) divided by $\mathrm{PCI}$ duration time in minutes.

The arbitrary cut-off value for $\mathrm{HL}$ of $\geq 2 \mathrm{~mm} \mathrm{Hg} / \mathrm{min}$ was chosen as the first condition of a significant hypotension episode. Further, we decided that a MAP decrease of at least $40 \%$ of PSAHT at any time during the $\mathrm{PCl}$ (maximal hypotension episode $(\mathrm{MHEP})=$ (minimum MAP - PSAHT/PSAHT) $\times 100 \%$ ) also qualifies as a significant hypotension episode (second condition). Lastly, we took in consideration the pure clinical definition of haemodynamic instability (third condition), namely when hypotension required intervention (e.g. fluid bolus, administration of catecholamines, intubation, defibrillation, resuscitation, or crossover to salvage IABP therapy). All of above-mentioned conditions ( 1 to 3 ) together formed our composite haemodynamic endpoint.

The study protocol was approved by the Local Bioethics Committee.

\section{Statistical analysis}

We performed primary intension-to-treat (ITT) analysis for 36 randomised patients and secondary per-treatment (PT) analysis for all 47 patients (randomised and from the registry).

Categorical variables were presented as counts and percentages, and continuous variables were presented as mean with standard deviation or median with the first and the third quartile as appropriate. Normality was assessed using the Shapiro-Wilk test. Equality of variances was tested using the Levene test. Comparisons of continuous variables were preformed using analysis of variance or Kruskal-Wallis test as appropriate. Post hoc analysis was performed using Tukey's HSD or the Steel-Dwass test, as appropriate. Paired analysis was performed using the mixed effect model. Pearson's or Spearman's correlation coefficients were calculated, as appropriate, based on the normality of the data. Fisher's exact test or the $\chi^{2}$ test were used to compare distributions of nominal variables. Simple logistic regression models were used to calculate odds ratios for the composite endpoints. A multiple logistic regression model was then constructed. All potential predictors were screened. All variables for which simple models showed a $p$-value less than 0.15 were candidates for the multiple model. A stepwise approach was used and minimisation of a Bayesian information criterion (BIC) was used as a target. The type of balloon used was locked in the model. C-statistic was calculated as a measure of goodness of fit. Bootstrap validation was performed with 1000 iterations. Variance inflation factors (VIF) were calculated to measure collinearity.

All tests were two-sided, and $p<0.05$ was assumed to indicate statistical significance. All data management and analysis activities were performed using JMP 14.2 (2019, SAS Institute Inc., Cary, NC, USA) and R 3.5.3 (R Core Team [2019]) [15].

\section{Results}

In the study period we were able to screen $47 \mathrm{pa}$ tients, 36 of whom were randomised: 13 in MEGA, 14 in STRD, and 9 in the CTRL group. Four patients in the CTRL group and one patient in the MEGA group did not receive the allocated intervention because of severely angulated and/or calcified femoral/iliac arteries. Eleven patients were screening failures but finally received one of the analysed treatments (MEGA, STRD, CTRL) and formed a registry (REG). Additional per treatment (PT) analysis was performed to control for cross-over and after adding REG patients, which included 47 subjects: 21 in the CTRL, 10 in the STRD, and 16 in the MEGA group (Figure 1).

The clinical data for randomised patients is shown in Table I. Almost all analysed parameters were equally distributed between the study subgroups, with the exception of more frequent diagnosis of ACS and slightly greater EF in the MEGA group. The randomised patients were characterised by a very high-risk profile with a mean $\mathrm{EF}$ of $30 \%$, median Syntax Score of 38 points, median Euroscore II mortality risk of $6 \%$, and median BCIS-1 Jeopardy Score of 12.0. These baseline characteristics were also similar in PT analysis with no difference between the groups, apart from more frequent peripheral artery disease in the CTRL group (Table II).

There was no difference in in-hospital and follow-up MACE incidence on ITT analysis for randomised patients: MACE during hospitalisation was observed in $23.1 \%$ of patients in the MEGA group, $7.1 \%$ of the STRD group, and $33.3 \%$ of the CTRL group $(p=0.25)$ and at FU in $50.0 \%$, $35.7 \%$, and $55.6 \%$, respectively $(p=0.61)$. Also, we did not observe any difference in safety endpoints: major or minor haemorrhagic complications, acute renal failure, or vascular complications (Table III).

The most frequent causes of major bleeds were: large haematoma at the vascular access site $(n=3)$, bleeding at the site of intravascular catheter without haematoma formation $(n=2)$, coronary artery perforation with cardiac tamponade $(n=2)$, significant haemoglobin drop without obvious cause $(n=2)$, gastrointestinal bleeding 


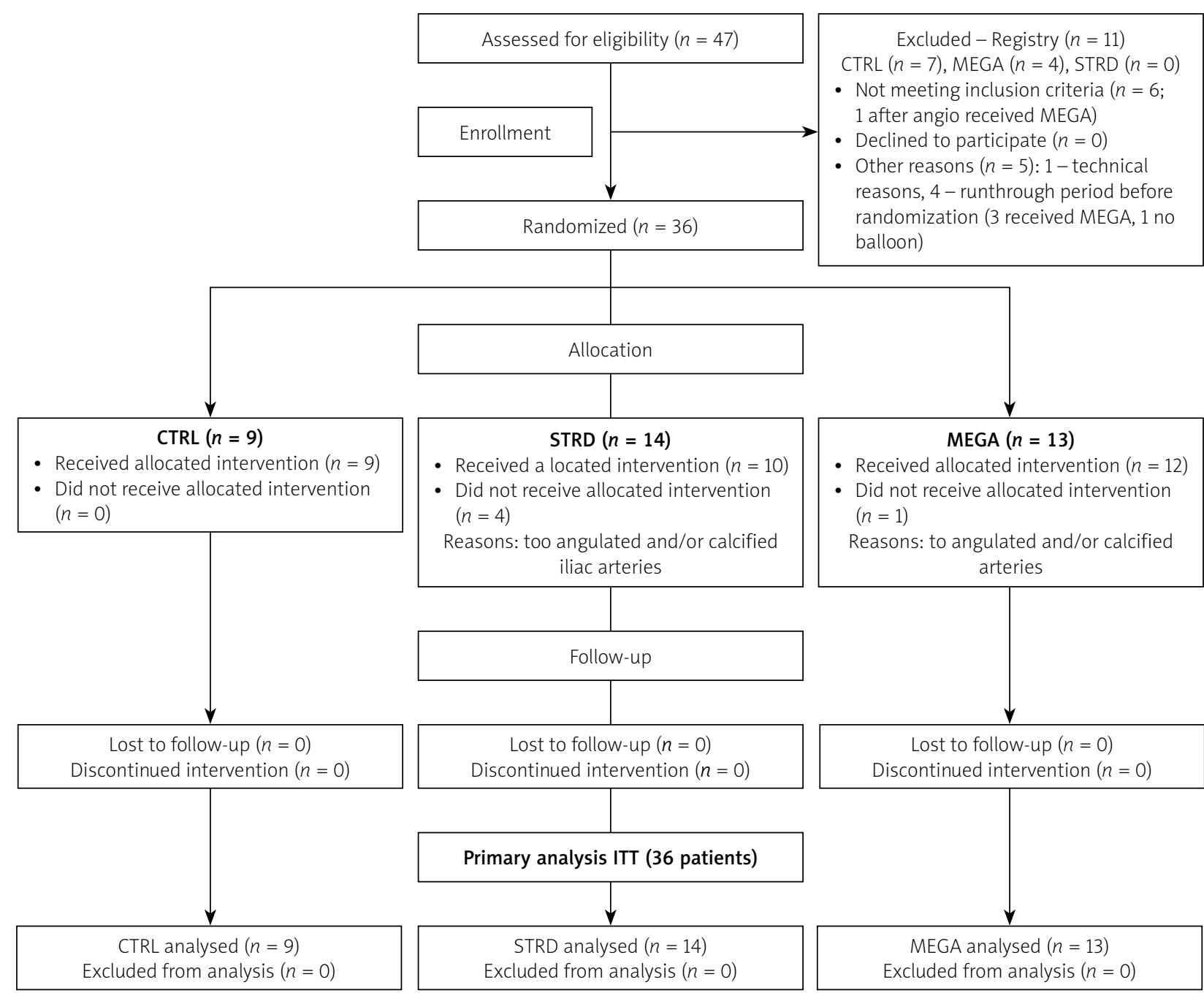

Secondary analysis PT (all 47 patients)

$\operatorname{CTRL}(n=21)(9+7+5)$

Excluded from analysis $(n=0)$
$\operatorname{STRD}(n=10)$

Excluded from analysis $(n=0)$
$\operatorname{MEGA}(n=16)(12+4)$

Excluded from analysis $(n=0)$

Figure 1. Study flow chart

$(n=1)$, alveolar haemorrhage $(n=1)$, and vascular surgical intervention $(n=1)$.

In ITT analysis composite haemodynamic endpoint was achieved in 2 of 13 (15.4\%) patients in MEGA, in 7 of $14(50.0 \%)$ patients in STRD, and in 4 of $9(44.4 \%)$ patients in the CTRL group, which potentially indicates clinical relevance, although no statistical significance was achieved $(p=0.12)$. One patient in the CTRL group required urgent cross-over to IABP therapy due to shock (Table IV).

Due to the occurrence of crossovers a PT set was formed, consisting of 16 patients in the MEGA, 10 in the STRD, and 21 in the CTRL group, including 11 patients initially assigned to REG, who finally received one of an- alysed treatments (MEGA, STRD, CTRL). In PT analysis there was no difference in study endpoints: MACE during hospitalisation was observed in $18.8 \%$ of patients in the MEGA group, $10.0 \%$ of the STRD group, and $23.8 \%$ of the CTRL group ( $p=0.64)$, MACE at FU occurred in $53.3 \%, 20.0 \%$, and $57.1 \%(p=0.12)$, major bleeding in $37.5 \%, 20.0 \%$, and $33.3 \%(p=0.62)$, and minor bleeding in $12.5 \%, 30.0 \%$, and $9.52 \%$, respectively $(p=0.35)$ (Table V).

Conversely, in PT analysis, we found a significant difference between the study subgroups for the occurrence of the composite haemodynamic endpoint (MEGA $12.5 \%$, STRD 50.0\%, and CTRL 52.4\%; $p=0.02$ ), although its individual components were not different (Table VI). 
Table I. Clinical, demographic, echocardiographic, angiographic, and procedural data (ITT)

\begin{tabular}{|c|c|c|c|c|}
\hline Variable & MEGA & STRD & CTRL & Total \\
\hline$N(\%)$ & $13(36)$ & $14(38)$ & $9(25)$ & $36(100)$ \\
\hline \multicolumn{5}{|l|}{ Demographic data: } \\
\hline Age [years], mean $\pm S D$ & $71.9 \pm 8.3$ & $73.3 \pm 11.2$ & $67.4 \pm 10.5$ & $71.3 \pm 10.0$ \\
\hline Male sex & $11(84.6)$ & $11(78.6)$ & $8(88.9)$ & $30(83.3)$ \\
\hline ACS at presentation* & $10(76.9)$ & $5(35.7)$ & $3(33.3)$ & $18(50.0)$ \\
\hline \multicolumn{5}{|l|}{ Clinical symptoms: } \\
\hline CCS class 3/4 & $9(69.2)$ & $11(78.6)$ & $3(33.3)$ & $23(63.9)$ \\
\hline NYHA class 3/4 & $8(61.5)$ & $12(85.7)$ & $8(88.7)$ & $28(77.8)$ \\
\hline \multicolumn{5}{|l|}{ Past medical history: } \\
\hline Hypertension & $13(100.0)$ & $14(100.0)$ & 9 (100.0) & $36(100.0)$ \\
\hline Diabetes & $4(30.8)$ & $9(64.3)$ & $4(44.4)$ & $17(47.2)$ \\
\hline Smoking & $7(58.3)$ & $10(71.4)$ & $7(77.8)$ & $24(68.6)$ \\
\hline Previous MI & $6(46.2)$ & $9(64.3)$ & $7(77.8)$ & $22(61.1)$ \\
\hline Previous PCl & $4(30.8)$ & $6(42.7)$ & $3(33.3)$ & $13(36.1)$ \\
\hline Previous CABG & $1(7.7)$ & $2(14.3)$ & $1(11.1)$ & $4(11.1)$ \\
\hline Previous stroke & $2(15.4)$ & $1(7.1)$ & $4(44.4)$ & $7(19.4)$ \\
\hline Heart failure & $7(53.9)$ & $10(71.4)$ & $8(88.9)$ & $25(69.4)$ \\
\hline Atrial fibrillation & $2(15.4)$ & $6(42.9)$ & $3(33.3)$ & $11(30.6)$ \\
\hline Hypercholesterolaemia & $11(84.6)$ & $10(71.4)$ & $6(66.7)$ & $27(75.0)$ \\
\hline Chronic kidney disease & $4(30.8)$ & $3(21.4)$ & $3(33.3)$ & $10(27.8)$ \\
\hline Peripheral artery disease & $4(30.8)$ & $3(21.4)$ & $4(44.4)$ & $11(30.6)$ \\
\hline \multicolumn{5}{|l|}{ Echo examination: } \\
\hline $\mathrm{EF}(\%)$, mean $\pm S D^{\star *}$ & $35 \pm 12$ & $29 \pm 10$ & $25 \pm 7$ & $30 \pm 11$ \\
\hline Significant mitral regurg. & $6(46.2)$ & $5(35.7)$ & $4(44.4)$ & $15(41.7)$ \\
\hline \multicolumn{5}{|l|}{ Risk scales: } \\
\hline SYNTAX score, median (IQR) & $42.0(30.0 ; 52.0)$ & $38.5(29.6 ; 47.1)$ & $36.3(24.5 ; 42.8)$ & $38.0(29.0 ; 47.0)$ \\
\hline EUROSCORE II (\%), median (IQR) & $6.0(3.0 ; 14.0)$ & $7.0(3.0 ; 13.0)$ & $5.0(3.0 .0 ; 19.0)$ & $6.0(3.0 ; 14.0)$ \\
\hline BCIS-1 Jeopardy score, median (IQR) & $12.0(10.0 ; 12.0)$ & $12.0(9.5 ; 12.0)$ & $10.0(8.0 ; 12.0)$ & $12.0(8.0 ; 12.0)$ \\
\hline \multicolumn{5}{|l|}{ Angiographic data: } \\
\hline Left main stenosis & $8(61.5)$ & $9(64.3)$ & $5(55.6)$ & $22(61.1)$ \\
\hline CTO present & $9(69.2)$ & $12(85.7)$ & $7(77.8)$ & $28(77.8)$ \\
\hline PCI MVD ( $\geq 2$ vessels) & $10(76.9)$ & $10(71.4)$ & $7(77.8)$ & $27(75.0)$ \\
\hline Radiation [GY], mean \pm SD & $2.4 \pm 1.6$ & $2.3 \pm 1.4$ & $2.7 \pm 1.7$ & $2.4 \pm 1.5$ \\
\hline Contrast vol. [ml], mean \pm SD & $317 \pm 94$ & $313 \pm 83$ & $293 \pm 86$ & $310 \pm 86$ \\
\hline No. of stents, median (IQR) & $2.0(1.15 ; 3.0)$ & $2.0(1.8 ; 2.3)$ & $2.0(1.0 ; 3.0)$ & $2.0(1.3 ; 3.0)$ \\
\hline Rotablation & $3(23.1)$ & $4(28.6)$ & $1(11.1)$ & $8(22.2)$ \\
\hline IVUS & $2(15.4)$ & $5(35.7)$ & $2(22.2)$ & $9(25.0)$ \\
\hline
\end{tabular}

Data are presented as number and percentage or mean value with standard deviation or median with interquartile range; ${ }^{*} p=0.05,{ }^{* *} p=0.03$.

In addition, patients who had had haemodynamic instability (assessed by a positive haemodynamic composite endpoint) were more likely to have periprocedural $\mathrm{MI}$ than patients who did not experience instability $(33.3 \%$ vs. $3.4 \%, p=0.01)$, with no difference in incidence of acute kidney injury (16.7\% vs. 6.9\%, $p=0.29)$.

Finally, on the PT cohort, we performed simple and multiple analyses for the occurrence of composite haemodynamic endpoint. In a simple model we found significant correlations with end diastolic volume (EDV), syntax score, left main (LM) disease, $\mathrm{PCl}$ of $\mathrm{LM}$, and $\mathrm{PCl}$ with rotablation and $\mathrm{PCl}$ complications. Then, using multiple regression analysis, we created a multivariable model that showed that PCI with MEGA balloon in comparison to STRD or CTRL diminished the risk of haemodynamic composite endpoint and, at the same time, performing $\mathrm{PCl}$ of the left main coronary artery or rotational atherectomy increased that risk. The C-statistic for the final multiple model was 0.889 , and the bootstrap C-statistic was 0.828 , which indicates a strong model. All VIFs were smaller than 2 (the highest for rotablation - 1.39, and the lowest for left main - 1.22).

Cardiac index measured pre-procedurally in this model did not have any influence on haemodynamic instability risk (Table VII). 
Table II. Clinical, demographic, echocardiographic, angiographic, and procedural data (PT)

\begin{tabular}{|c|c|c|c|c|}
\hline Variable & MEGA & STRD & CTRL & Total \\
\hline$N(\%)$ & $16(34)$ & $10(21)$ & $21(45)$ & $47(100)$ \\
\hline \multicolumn{5}{|l|}{ Demographic data: } \\
\hline Age [years], mean \pm SD & $71.4 \pm 8.4$ & $71.3 \pm 11.5$ & $71.7 \pm 10.1$ & $71.5 \pm 9.7$ \\
\hline Male sex & $13(81.3)$ & $8(80.0)$ & $18(85.7)$ & 39 (82.9) \\
\hline ACS at presentation & $11(68.8)$ & $3(30.0)$ & $8(38.1)$ & $22(46.8)$ \\
\hline \multicolumn{5}{|l|}{ Clinical symptoms: } \\
\hline CCS class $3 / 4$ & $10(62.5)$ & $7(70.0)$ & $10(47.6)$ & $27(57.5)$ \\
\hline NYHA class 3/4 & $11(68.8)$ & $8(80.0)$ & $14(66.7)$ & $33(70.2)$ \\
\hline \multicolumn{5}{|l|}{ Past medical history: } \\
\hline Hypertension & $16(100.0)$ & $10(100.0)$ & $20(95.2)$ & $46(97.9)$ \\
\hline Diabetes & $7(43.8)$ & $6(60.0)$ & $10(47.6)$ & $23(48.9)$ \\
\hline Smoking & $8(53.3)$ & $8(80.0)$ & $16(76.2)$ & $32(69.6)$ \\
\hline Previous MI & $8(50.0)$ & $6(60.0)$ & $13(61.9)$ & $27(57.5)$ \\
\hline Previous PCl & $5(31.3)$ & $4(40.0)$ & $7(33.3)$ & $16(34.0)$ \\
\hline Previous CABG & $2(12.5)$ & $1(10.0)$ & $2(9.5)$ & $5(10.6)$ \\
\hline Previous stroke & $2(12.5)$ & $0(0.0)$ & $6(28.6)$ & $8(17.0)$ \\
\hline Heart failure & $10(62.5)$ & $6(60.0)$ & $16(76.2)$ & $32(68.1)$ \\
\hline Atrial fibrillation & $5(31.3)$ & $5(50.0)$ & $6(28.6)$ & $16(34.0)$ \\
\hline Hypercholesterolaemia & $13(81.3)$ & $6(60.0)$ & $15(71.4)$ & $34(72.3)$ \\
\hline Chronic kidney disease & $7(43.8)$ & $1(10.0)$ & $5(23.8)$ & $13(27.7)$ \\
\hline Peripheral artery disease* & $6(37.5)$ & $0(0.0)$ & $11(52.4)$ & $17(36.1)$ \\
\hline \multicolumn{5}{|l|}{ Echo examination: } \\
\hline $\mathrm{EF}(\%)$, mean $\pm \mathrm{SD}$ & $33 \pm 9$ & $29 \pm 11$ & $33 \pm 13$ & $32 \pm 11$ \\
\hline Significant mitral regurg. & $8(50.0)$ & $4(40.0)$ & $8(38.1)$ & $20(42.6)$ \\
\hline \multicolumn{5}{|l|}{ Risk scales: } \\
\hline SYNTAX score, median (IQR) & $36.5(29.1 ; 49.6)$ & $38.5(29.6 ; 43.0)$ & $36.3(27.8 ; 45.5)$ & $38.0(29.0 ; 44.5)$ \\
\hline EUROSCORE II (\%), median (IQR) & $8(4 ; 14)$ & $6(2 ; 8)$ & $5(4 ; 15)$ & $6(3 ; 12)$ \\
\hline BCIS-1 Jeopardy score, median (IQR) & $12.0(9.0 ; 12.0)$ & $12.0(10.0 ; 12.0)$ & $12.0(8.0 ; 12.0)$ & $12.0(8.0 ; 12.0)$ \\
\hline \multicolumn{5}{|l|}{ Angiographic data: } \\
\hline Left main stenosis & $11(68.8)$ & $6(60.00)$ & $15(71.43)$ & $32(68.09)$ \\
\hline CTO present & $12(75.0)$ & $9(90.00)$ & $17(80.95)$ & $38(80.85)$ \\
\hline PCI MVD ( $\geq 2$ vessels) & $13(81.3)$ & $7(70.00)$ & $17(80.95)$ & $37(78.72)$ \\
\hline Radiation [GY], mean \pm SD & $2.4 \pm 1.5$ & $2.3 \pm 1.5$ & $2.3 \pm 1.3$ & $2.3 \pm 1.4$ \\
\hline Contrast vol. [ml], mean \pm SD & $317 \pm 89$ & $310 \pm 94$ & $295 \pm 71$ & $305 \pm 81$ \\
\hline No. of stents, median (IQR) & $2.0(1.0 ; 3.0)$ & $2.0(1.0 ; 2.3)$ & $2.0(1.5 ; 2.5)$ & $2.0(1.0 ; 3.0)$ \\
\hline Rotablation & $2(12.5)$ & $3(30.0)$ & $5(23.8)$ & $10(21.3)$ \\
\hline IVUS & $3(18.8)$ & $5(50.0)$ & $5(23.8)$ & $13(27.7)$ \\
\hline
\end{tabular}

Data are presented as number and percentage or mean value with standard deviation or median with interquartile range; ${ }^{*} p=0.0036$.

\section{Discussion}

The goal of circulatory support in a high-risk PCI setting is to maintain a stable haemodynamic state throughout the intervention by altering the myocardial ischaemic threshold and allowing time for complex procedure, especially when complications (embolisation, dissection, abrupt vessel closure, etc.) develop. Therefore, theoretically, the best cardiac assist device would reduce myocardial oxygen demand (via reductions in myocardial wall tension) and simultaneously increase oxygen supply by augmenting diastolic pressure and coronary blood flow. On the other hand, it should be easily percutane- ously implanted and small enough to decrease the risk of vascular complications [16].

The present study is, to our knowledge, the only randomised study comparing larger volume IABP with standard volume or no balloon support during HRPCI. Unfortunately, we could not show any difference between the groups in ITT analysis for the occurrence of MACE during hospitalisation and at 1-year follow-up. This was also true in PT analysis replicating the results of much larger studies, such as BICIS-1, which recruited patients to elective standard volume IABP placement before $\mathrm{HRPCI}$ or to a no-balloon control group. The reported 30-day MACE 
Table III. In-hospital and follow-up MACE and safety endpoints (ITT)

\begin{tabular}{|c|c|c|c|c|c|}
\hline Variable & MEGA & STRD & CTRL & Total & $P$-value \\
\hline$N(\%)$ & $13(36)$ & $14(38)$ & $9(25)$ & $36(100)$ & \\
\hline In-hospital MACE: & $3(23.08)$ & $1(7.14)$ & $3(33.33)$ & 7 (19.44) & 0.25 \\
\hline Cardio-vascular death & $1(7.69)$ & $0(0.00)$ & $0(0.00)$ & $1(2.78)$ & 0.35 \\
\hline Myocardial infarction & $2(15.38)$ & $1(7.14)$ & $2(22.22)$ & $5(13.89)$ & 0.57 \\
\hline Re-PCl & $0(0.00)$ & $1(7.14)$ & $0(0.00)$ & $1(2.78)$ & 0.38 \\
\hline Exacerbation of heart failure & $1(7.69)$ & $0(0.00)$ & $0(0.00)$ & $1(2.78)$ & 0.35 \\
\hline Stroke & $0(0.00)$ & $0(0.00)$ & $1(11.11)$ & $1(2.78)$ & 0.24 \\
\hline Follow-up MACE: & $6(50.00)$ & $5(35.71)$ & $5(55.56)$ & $16(45.71)$ & 0.60 \\
\hline Cardio-vascular deaths & $2(16.67)$ & $2(14.29)$ & $4(44.44)$ & $8(22.86)$ & 0.23 \\
\hline Deaths (other reasons) & $1(8.33)$ & $1(7.14)$ & $0(0.00)$ & $2(5.71)$ & 0.54 \\
\hline Myocardial infarction/RE-PCI & $2(16.67)$ & $0(0.00)$ & $1(11.11)$ & $3(8.57)$ & 0.18 \\
\hline Exacerbation of heart failure & $2(16.67)$ & $3(21.43)$ & $3(33.33)$ & $8(22.86)$ & 0.67 \\
\hline Stroke & $1(8.33)$ & $0(0.00)$ & $0(0.00)$ & $1(2.86)$ & 0.33 \\
\hline \multicolumn{6}{|l|}{ In-hospital safety enpoints: } \\
\hline Major haemorrhages (BARC $\geq 3$ ) & $6(46.15)$ & $4(28.57)$ & $2(22.22)$ & $12(33.33)$ & 0.45 \\
\hline Minor haemorrhages $($ BARC $<3)$ & $2(15.38)$ & $3(21.43)$ & $2(22.22)$ & 7 (19.44) & 0.89 \\
\hline \multicolumn{6}{|l|}{ Vascular access complications: } \\
\hline Haematoma & $1(7.69)$ & $4(28.57)$ & $2(22.22)$ & 7 (19.44) & 0.34 \\
\hline Aneurism & $0(0.00)$ & $0(0.00)$ & $0(0.00)$ & $0(0.00)$ & - \\
\hline A-V fistula & $1(7.69)$ & $0(0.00)$ & $0(0.00)$ & $1(2.78)$ & 0.35 \\
\hline Acute ischaemia & $2(15.38)$ & $0(0.00)$ & $0(0.00)$ & $2(5.56)$ & 0.12 \\
\hline Infection & $1(7.69)$ & $0(0.00)$ & $0(0.00)$ & $1(2.78)$ & 0.35 \\
\hline Acute renal failure & $2(15.38)$ & $1(7.14)$ & $0(0.00)$ & $3(8.33)$ & 0.32 \\
\hline
\end{tabular}

Table IV. Composite haemodynamic endpoint (ITT)

\begin{tabular}{|c|c|c|c|c|c|}
\hline Variable & MEGA & STRD & CTRL & Total & $P$-value \\
\hline$N(\%)$ & $13(36)$ & $14(38)$ & $9(25)$ & $36(100)$ & \\
\hline Composite haemodynamic endpoint: & $2(15.38)$ & $7(50.00)$ & $4(44.44)$ & $13(36.11)$ & 0.12 \\
\hline $\mathrm{HL}[\mathrm{mm} \mathrm{Hg} / \mathrm{min}]:$ & & & & & 0.44 \\
\hline Mean \pm SD & $0.7 \pm 1.5$ & $2.6 \pm 3.2$ & $1.7 \pm 2.4$ & $1.6 \pm 2.5$ & \\
\hline Median (IQR) & $0.1(0.00 ; 0.3)$ & $1.6(0.0 ; 4.4)$ & $0.8(0.04 ; 2.7)$ & $0.14(0.0 ; 2.5)$ & \\
\hline MHEP (\%): & & & & & 0.20 \\
\hline Mean \pm SD & $-19 \pm 18$ & $-34 \pm 26$ & $-32 \pm 19$ & $-28 \pm 22$ & \\
\hline Median (IQR) & $-17(-25 ;-8)$ & $-34(-61 ;-8)$ & $-24(-48 ;-15)$ & $-22(-42 ;-11)$ & \\
\hline Clinical instability & $1(7.69)$ & $4(28.57)$ & $2(22.22)$ & $7(19.44)$ & 0.34 \\
\hline
\end{tabular}

HL - hypotension load, MHEP - maximal hypotension episode.

rate in this trial was $15.2 \%$ in IABP and $16.0 \%$ in CTRL patients $(p=0.85)$ [4]. It needs to be stressed, however, that after 5 years of FU in the BICIS- 1 trial the mortality rate in elective IABP patients was less than in the CTRL group (OR $=0.66,95 \% \mathrm{Cl}: 0.44-0.98 ; p=0.039)$ [17]. Some metanalyses also suggest that there could be mortality benefit of elective IABP during HRPCI [18], but some others did not [19]. The debate is still ongoing whether more potent devices such as the Impella percutaneous axial heart pump (Abiomed, Danvers, MA) should substitute IABP. To date, there is only one randomised trial of Impella 2.5 vs. IABP (standard volume) in an $\mathrm{HRPCl}$ setting - the PROTECT II study [20], which failed to meet its primary 30-day composite end-point of reducing MACE in primary ITT analysis (30-day MACE Impella $35.1 \%$ vs. IABP $42.2 \%, p=0.092$ ), although secondary PT analysis showed superiority of the more potent device (90-day MACE Impella $40.0 \%$ vs. IABP $51.0 \%, p=0.023$ ). On the other hand, new registry studies raise further doubts on the percutaneous pump, showing higher rates of bleeding, stroke, and even death in both HRPCl and shock indication [21]. Last but not least, there is fresh evidence in favour of IABP - it has recently been shown that $50 \mathrm{ml} \mathrm{IABP}$ outperformed inotropes (enoximone or dobutamine) in acute heart failure patients without acute coronary syndrome [22].

Our study did not show a difference in the rate of major or minor bleedings according to BARC definitions be- 
Table V. In-hospital and follow-up MACE and safety endpoints (PT)

\begin{tabular}{|c|c|c|c|c|c|}
\hline Variable & MEGA & STRD & CTRL & Total & $P$-value \\
\hline$N(\%)$ & $16(34)$ & $10(21)$ & $21(45)$ & $47(100)$ & \\
\hline In-hospital MACE: & $3(18.75)$ & $1(10.00)$ & $5(23.81)$ & 9 (19.15) & 0.63 \\
\hline Cardio-vascular death & $1(6.25)$ & $0(0.00)$ & $0(0.00)$ & $1(2.13)$ & 0.33 \\
\hline Myocardial infarction & $2(12.50)$ & $1(10.00)$ & $4(19.05)$ & 7 (14.89) & 0.76 \\
\hline $\mathrm{Re}-\mathrm{PCl}$ & $0(0.00)$ & $1(10.00)$ & $0(0.00)$ & $1(2.13)$ & 0.20 \\
\hline Exacerbation of heart failure & $1(6.25)$ & $0(0.00)$ & $0(0.00)$ & $1(2.13)$ & 0.35 \\
\hline Stroke & $0(0.00)$ & $0(0.00)$ & $1(4.76)$ & $1(2.13)$ & 0.33 \\
\hline Follow-up MACE: & $8(53.33)$ & $2(20.00)$ & $12(57.14)$ & $22(47.83)$ & 0.12 \\
\hline Cardio-vascular deaths & $3(20.00)$ & $2(20.00)$ & $4(19.05)$ & $9(19.57)$ & 0.99 \\
\hline Deaths (other reasons) & $1(6.67)$ & $0(0.00)$ & $4(19.05)$ & $5(10.87)$ & 0.14 \\
\hline Myocardial infarction/RE-PCI & $3(20.00)$ & $0(0.00)$ & $1(4.76)$ & $4(8.70)$ & 0.13 \\
\hline Exacerbation of heart failure & $2(13.33)$ & $1(10.00)$ & $6(28.57)$ & $9(19.57)$ & 0.36 \\
\hline Stroke & $1(6.67)$ & $0(0.00)$ & $1(4.76)$ & $2(4.35)$ & 0.59 \\
\hline \multicolumn{6}{|l|}{ In-hospital safety endpoints: } \\
\hline Major haemorrhages (BARC $\geq 3$ ) & $6(37.50)$ & $2(20.00)$ & $7(33.33)$ & $15(31.91)$ & 0.62 \\
\hline Minor haemorrhages $($ BARC $<3)$ & $2(12.50)$ & $3(30.00)$ & $2(9.52)$ & 7 (14.89) & 0.35 \\
\hline \multicolumn{6}{|l|}{ Vascular access complications: } \\
\hline Haematoma & $1(6.25)$ & $3(30.00)$ & $3(14.29)$ & 7 (14.89) & 0.27 \\
\hline Aneurism & $0(0.00)$ & $0(0.00)$ & $0(0.00)$ & $0(0.00)$ & - \\
\hline A-V fistula & $1(6.25)$ & $0(0.00)$ & $0(0.00)$ & $1(2.13)$ & 0.33 \\
\hline Acute ischaemia & $2(12.50)$ & $0(0.00)$ & $0(0.00)$ & $2(4.26)$ & 0.11 \\
\hline Infection & $1(6.25)$ & $0(0.00)$ & $0(0.00)$ & $1(2.13)$ & 0.33 \\
\hline Acute renal failure & $1(6.25)$ & $0(0.00)$ & $2(9.52)$ & $3(6.38)$ & 0.44 \\
\hline
\end{tabular}

Table VI. Composite haemodynamic endpoint (PT)

\begin{tabular}{|c|c|c|c|c|c|}
\hline Variable & MEGA & STRD & CTRL & Total & $P$-value \\
\hline$N(\%)$ & $16(34)$ & $10(21)$ & $21(45)$ & $47(100)$ & \\
\hline Composite haemodynamic endpoint: & $2(12.5)$ & $5(50.0)$ & $11(52.4)$ & $18(38.3)$ & 0.02 \\
\hline $\mathrm{HL} \underline{[\mathrm{mm} \mathrm{Hg} / \mathrm{min}]:}$ & & & & & 0.16 \\
\hline Mean \pm SD & $0.7 \pm 1.4$ & $2.1 \pm 2.6$ & $2.4 \pm 3.1$ & $1.7 \pm 2.6$ & \\
\hline Median (IQR) & $0.09(0.0 ; 0.4)$ & $1.2(0.0 ; 4.4)$ & $1.2(0.1 ; 2.5)$ & $0.3(0.0 ; 2.5)$ & \\
\hline MHEP (\%): & & & & & 0.15 \\
\hline Mean \pm SD & $-19 \pm 18$ & $-30 \pm 25$ & $-33 \pm 20$ & $-28 \pm 21$ & \\
\hline Median (IQR) & $-16(-24 ;-6)$ & $-32(-49 ;-6)$ & $-29(-50 ;-13)$ & $-23(-41 ;-11)$ & \\
\hline Clinical instability & $1(6.25)$ & $2(20.00)$ & $6(28.57)$ & $9(19.15)$ & 0.19 \\
\hline
\end{tabular}

HL - hypotension load, MHEP - maximal hypotension episode.

tween the study groups, similarly to the BICIS-1 study, as well as the risk of vascular and renal complications. The results of IABP-SHOCK II also confirm that IABP therapy is safe, with the same rate of major bleedings as in the control group. At the same time, as was said before, although more potent, the Impella pump, being larger in diameter than IABP, increases the incidence of vascular complications and the bleeding rate in comparison to IABP.

Because our study was not powered enough to access hard clinical outcomes, we compared the occurrence of significant hypotension between the groups as a secondary outcome. Unfortunately, there is no universal definition of severe intraprocedural hypotension. Some authors defined it as a decrease of mean arterial pressure below the cut-off point of $75 \mathrm{mmHg}$ for at least 10 min despite fluid resuscitation or requirement of inotropic support, as was used in the BCIS-1 trial. Others reported severe hypotension if systolic blood pressure or augmented diastolic pressure (the higher of the two) fell below $90 \mathrm{~mm} \mathrm{Hg}$ for $\geq 5$ min requiring inotropic/ pressor medication or IV fluids while on device support, as in the PROTECT II trail. For the purpose of this study we introduced novel hypotension indices such as hypotension load $(\mathrm{HL})$, which, as we described, captures all hypotension episodes during the $\mathrm{PCl}$ in relation to the baseline mean arterial pressure threshold value and is 
Table VII. Univariable and multivariable models for haemodynamic composite endpoint (PT)

\begin{tabular}{|c|c|c|c|c|}
\hline Variable & Odds ratio & Lower $95 \%$ & Upper $95 \%$ & $P$-value \\
\hline \multicolumn{5}{|l|}{ Univariable models } \\
\hline \multicolumn{5}{|l|}{ Parametric: } \\
\hline Age & 1.03 & 0.97 & 1.1 & 0.3467 \\
\hline EURO score II & 4.67 & 0 & 11868.53 & 0.7003 \\
\hline SYNTAX score & 1.06 & 0.99 & 1.13 & 0.0991 \\
\hline Jeopardy score & 1.06 & 0.77 & 1.45 & 0.7214 \\
\hline EF & 1.58 & 0.01 & 279.9 & 0.8622 \\
\hline Cardiac index (echo) & 0.91 & 0.36 & 2.3 & 0.8362 \\
\hline End diastolic volume & 0.99 & 0.98 & 1 & 0.0983 \\
\hline E/E' & 0.97 & 0.89 & 1.05 & 0.4602 \\
\hline Hs-TnT & 0.82 & 0.09 & 7.27 & 0.8622 \\
\hline CK-MB & 0.96 & 0.87 & 1.05 & 0.3423 \\
\hline NT-pro-BNP & 1 & 1 & 1 & 0.3667 \\
\hline Haemoglobin & 1.23 & 0.86 & 1.77 & 0.2577 \\
\hline No. of stents & 1.5 & 0.78 & 2.9 & 0.2246 \\
\hline Fluoro time & 1.01 & 0.97 & 1.05 & 0.5971 \\
\hline Contrast volume & 1 & 1 & 1.01 & 0.4628 \\
\hline Pre-PCI mean arterial pressure & 0.98 & 0.93 & 1.02 & 0.3524 \\
\hline Pulmonary capillary wedge pressure & 0.96 & 0.88 & 1.06 & 0.4157 \\
\hline Cardiac index (pulmonary artery cath) & 0.69 & 0.2 & 2.33 & 0.5488 \\
\hline Cardiac power output & 0.29 & 0.02 & 3.64 & 0.3342 \\
\hline $\mathrm{d} P / \mathrm{d} t$ & 1 & 1 & 1 & 0.9661 \\
\hline \multicolumn{5}{|l|}{ Non-parametric: } \\
\hline ACS & 0.56 & 0.17 & 1.85 & 0.3456 \\
\hline Male sex & 1.04 & 0.22 & 5.01 & 0.9594 \\
\hline Previous IM & 2.79 & 0.79 & 9.85 & 0.1118 \\
\hline Previous $\mathrm{PCl}$ & 1.41 & 0.41 & 4.85 & 0.5813 \\
\hline Previous CABG & 1.08 & 0.16 & 7.2 & 0.9340 \\
\hline Heart failure & 1.23 & 0.34 & 4.52 & 0.7537 \\
\hline Three- vs. two-vessel disease & 1.28 & 0.21 & 7.82 & 0.7892 \\
\hline Left main disease & 3.53 & 0.83 & 14.94 & 0.0868 \\
\hline CTO present & 2.55 & 0.47 & 13.91 & 0.2809 \\
\hline $\mathrm{PCl}$ left main & 6.5 & 1.26 & 33.58 & 0.0255 \\
\hline Rotablation & 5.52 & 1.2 & 25.35 & 0.0282 \\
\hline Multivessel PCI & 3.05 & 0.57 & 16.36 & 0.1937 \\
\hline PCl complications & 4.33 & 0.92 & 20.32 & 0.0629 \\
\hline MEGA vs. CTRL & 0.13 & 0.02 & 0.72 & 0.0194 \\
\hline VSTRD vs. CTRL & 0.91 & 0.20 & 4.10 & 0.9013 \\
\hline \multicolumn{5}{|l|}{ Multivariable model: } \\
\hline MEGA vs. STRD & 0.062 & 0.002 & 0.822 & 0.0342 \\
\hline MEGA vs. CTRL & 0.032 & 0.001 & 0.343 & 0.0025 \\
\hline PCl left main & 14.318 & 2.050 & 171.021 & 0.0057 \\
\hline Rotablation & 15.155 & 1.661 & 234.043 & 0.0147 \\
\hline $\mathrm{Cl}$ (echo) & 0.324 & 0.059 & 1.318 & 0.1192 \\
\hline
\end{tabular}

indexed for units of time and maximum hypotension episode (MHEP), which is the minimum pressure below the threshold value, which, together with pure clinical instability, formed our composite haemodynamic endpoint. In ITT analysis this new composite haemodynamic endpoint was close to reaching statistical significance, which potentially indicates clinical relevance. Conversely, in PT analysis, the observed difference was statistically significant, with the lowest incidence of haemodynamic instability in the MEGA group. Similarly, in the BICIS-1 tri- 
al there were fewer procedural complications in the IABP group $(1.3 \%$ vs. $10.7 \%, p<0.001)$, the majority of which were prolonged hypotension episodes in the no-elective IABP group (in $12 \%$ of cases requiring emergency IABP placement). On the other hand, in PROTECT II significant hypotension occurred in $9 \%$ of IABP patients and $4.0 \%$ of Impella patients, which was not statistically significant ( $p=0.07$ ), although cardiac power output (CPO) during $\mathrm{PCl}$ was better preserved with Impella than with IABP (maximal drop of CPO of $-0.04 \pm 0.24$ vs. $-0.14 \pm 0.27, p=$ 0.001 , respectively). But, as was said before, a standard volume balloon was used. It is then probable that even such a small additional balloon volume could have a substantial effect on hypotension risk. The haemodynamic profile of larger volume balloon was studied by Kapur et al., who demonstrated greater augmented diastolic blood pressure, greater systolic unloading (which were both linearly correlated), and a larger reduction of PCWP of $50 \mathrm{ml}$ balloon in respect to $40 \mathrm{ml}$ in both $\mathrm{HRPCl}$ and shock patients [7].

To account for cross-over and after including REG patients to the PT cohort, we subsequently performed multiple regression analysis, which showed that circulatory support with IABP of larger volume was independently correlated with diminished risk of composite haemodynamic endpoint (MEGA vs. STRD: OR $=0.062, p=0.034$ and MEGA vs. CTRL: OR $=0.032, p=0.002)$. There is evidence from a recently published paper from the anaesthesiological field that may be clinically relevant because the authors, after analysing various absolute and relative thresholds for hypotension, showed that a decrease of MAP below $65 \mathrm{~mm} \mathrm{Hg}$ or below 20\% from baseline were both related to myocardial injury after noncardiac surgery (MINS) and acute kidney injury (AKI). Moreover, at any given threshold prolonged exposure was associated with increased odds of risk. At a MAP of $50 \mathrm{~mm} \mathrm{Hg}$, for example, just one minute was enough to significantly increase the risk for both myocardial and renal injury [23]. In our study the composite endpoint of haemodynamic instability was shown to be correlated to in-hospital (periprocedural) myocardial infarction, but not with AKI.

It should be stressed that all of the IABP implantations in our study were done electively before $\mathrm{PCl}$ had started, which, according to various clinical studies, especially on AMICS, would be the preferred timing for MCS usage, instead of inserting it only after the procedure has been completed [24]. On the other hand, only one of our patients initially randomised to the CTRL group had to be crossed over to IABP strategy as a consequence of severe haemodynamic instability.

Finally, we also demonstrated that haemodynamic instability was more frequent if rotational atherectomy was used or $\mathrm{PCl}$ of the left main coronary artery was performed, which is in line with common thoughts. But, rather unexpectedly, we found that cardiac index or EF did not have a statistically significant influence on composite haemodynamic endpoint. We speculate that this observation could be explained by the fact that almost all of our patients had relatively low baseline EF (32 $\pm 11 \%)$ and $\mathrm{Cl}$ (mean: $2.6 \pm 0.68 \mathrm{l} / \mathrm{min}$ ), and these results could have been different if we had recruited less sick patients with greater $\mathrm{EF}$ and $\mathrm{Cl}$.

Our study has important limitations. It was designed as a randomised study, but due to the slow recruitment process (single-centre study) we were able to randomise only 36 patients during the study period (18 months). Thus, it was significantly insufficiently powered to show any difference in hard clinical endpoints and should be viewed only as a pilot study. Moreover, we observed some cross-over due to inability to implant the device when tortuous or calcified femoral and iliac vessels were discovered intra-procedurally. To account for this limitation, we decided to perform PT analysis and increase the number of patients adding subjects from the REG and calculate the secondary outcome of composite haemodynamic endpoint using multiple regression models. Moreover, our composite haemodynamic endpoint is novel and needs further investigation to show its clinical significance or lack thereof.

\section{Conclusions}

In this small RCT we demonstrated that elective IABP of larger volume type implanted electively before $\mathrm{HRPCI}$ was able to reduce composite haemodynamic endpoint during the procedure, but we could not show any improvement in hard clinical endpoints of MACE in ITT and PT analysis.

\section{Conflict of interest}

The authors declare no conflict of interest.

\section{References}

1. Chieffo A, Burzotta F, Pappalardo F, et al. Clinical expert consensus document on the use of percutaneous left ventricular assist support devices during complex high-risk indicated PCl: Italian Society of Interventional Cardiology Working Group Endorsed by Spanish and Portuguese Interventional Card. Int J Cardiol 2019; 293: 84-90.

2. Kantrowitz A, Tjønneland S, Freed PS, et al. Initial clinical experience with intraaortic balloon pumping in cardiogenic shock. JAMA 1968; 203: 113-8.

3. Thiele H, Zeymer U, Neumann FJ, et al. Intraaortic balloon support for myocardial infarction with cardiogenic shock. NEJM 2012; 367: 1287-96.

4. Perera D, Stables R, Thomas M, et al. Elective intra-aortic balloon counterpulsation during high-risk percutaneous coronary intervention: a randomized controlled trial. JAMA 2010; 304: 867-74.

5. Patel MR, Smalling RW, Thiele H, et al. Intra-aortic balloon counterpulsation and infarct size in patients with acute anterior myocardial infarction without shock: the CRISP AMI randomized trial. JAMA 2011; 306: 1329-37. 
6. Rihal CS, Naidu SS, Givertz MM, et al. 2015 SCAI/ACC/HFSA/STS Clinical Expert Consensus Statement on the Use of Percutaneous Mechanical Circulatory Support Devices in Cardiovascular Care (Endorsed by the American Heart Association, the Cardiological Society of India, and Sociedad Latino Americana de Cardiologia Intervencion; Affirmation of Value by the Canadian Association of Interventional Cardiology-Association Canadienne de Cardiologie D'intervention). Catheter Cardiovasc Interv 2015; 85: E175-96.

7. Kapur NK, Paruchuri V, Majithia A, et al. Hemodynamic effects of standardversus larger-capacity intraaortic balloon counterpulsation pumps. J Invasive Cardiol 2015; 4: 182-8.

8. Urbaniak GC, Plous S. Research Randomizer (Version 4.0) [Computer software]. Retrieved on June 22, 2013, from http://www. randomizer.org/.

9. Windecker S, Kolh P, Alfonso F, et al. 2014 ESC/EACTS Guidelines on myocardial revascularization: The Task Force on Myocardial Revascularization of the European Society of Cardiology (ESC) and the European Association for Cardio-Thoracic Surgery (EACTS). Developed with the special contribution of the European Association of Percutaneous Cardiovascular Interventions (EAPCI). Eur Heart J 2014; 35: 2541-619.

10. Mehran R, Rao SV, Bhatt DL, et al. Standardized bleeding definitions for cardiovascular clinical trials: a consensus report from the bleeding academic research consortium. Circulation 2011; 123: 2736-47.

11. Perera D, Stables R, Booth J, et al. The Balloon pump-assisted Coronary Intervention Study (BCIS-1): rationale and design. Am Heart J 2009; 158: 910-6.

12. Thygesen K, Alpert JS, Jaffe AS, et al. Fourth universal definition of myocardial infarction. Eur Heart J 2019; 40: 237-69.

13. http://www.euroscore.org/calc.html

14. http://www.syntaxscore.com/calculator/start.htm

15. R Core Team (2018). R: A language and environment for statistical computing. R Foundation for Statistical Computing, Vienna, Austria. Available online at https://www.R-project.org/

16. Burkhoff D, Naidu SS. The science behind percutaneous hemodynamic support: a review and comparison of support strategies. Catheter Cardiovasc Interv 2012; 80: 816-29.

17. Perera D, Stables R, Clayton T, et al. Long-term mortality data from the balloon pump-assisted coronary intervention study (BCIS-1): a randomized, controlled trial of elective balloon counterpulsation during high-risk percutaneous coronary intervention. Circulation 2013; 127: 207-12.

18. Chen S, Yin Y, Ling Z, Krucoff MW. Short and long term effect of adjunctive intra-aortic balloon pump use for patients undergoing high risk reperfusion therapy: a meta-analysis of 10 international randomised trials. Heart 2014; 100: 303-10.

19. Wan YD, Sun TW, Kan QC, et al. The effects of intra-aortic balloon pumps on mortality in patients undergoing high-risk coronary revascularization: a meta-analysis of randomized controlled trials of coronary artery bypass grafting and stenting era. PLoS One 2016; 11: e0147291.

20. O'Neill WW, Kleiman NS, Moses J, et al. A prospective, randomized clinical trial of hemodynamic support with impella 2.5 versus intra-aortic balloon pump in patients undergoing high-risk percutaneous coronary intervention: the PROTECT II study. Circulation 2012; 126: 1717-27.

21. Amin AP, Spertus JA, Curtis JP, et al. The evolving landscape of Impella ${ }^{\circledR}$ use in the united states among patients undergoing percutaneous coronary intervention with mechanical circulatory support. Circulation 2020; 141: 273-84.

22. Den Uil CA, Van Mieghem NM, B Bastos M, et al. Primary intra-aortic balloon support versus inotropes for decompensated heart failure and low output: a randomised trial. Eurolntervention 2019; 15: 586-93.

23. Salmasi V, Maheshwari K, Yang D, et al. Relationship between intraoperative hypotension, defined by either reduction from baseline or absolute thresholds, and acute kidney and myocardial injury after noncardiac surgery. Anesthesiology 2017; 126: 47-65.

24. O'Neill WW, Schreiber T, Wohns DH, et al. The current use of Impella 2.5 in acute myocardial infarction complicated by cardiogenic shock: results from the USpella Registry. J Interv Cardiol 2014; 27: 1-11. 